\title{
EDITORIAL
}

\section{Short tandem repeat markers in diagnostics: what's in a repeat?}

Leukemia (2006) 20, 1353-1355. doi:10.1038/sj.leu.2404273

Short tandem repeats (STRs) represent intron polymorphism among individuals that occur frequently in the human genome. They consist of tandemly arranged nucleotide repeat units. Based upon a unique combination of STR alleles, individuals can be unequivocally identified. ${ }^{1}$ The number of STR markers required for unique characterization of individuals can be predicted based on the allele frequencies in a population.

The most common approach to identify STR alleles includes the in vitro amplification of STR (microsatellite) loci by the polymerase chain reaction (PCR) using primers flanking the repeat region and subsequent fragment analysis to identify the allele sizes. ${ }^{2-5}$

STR marker systems are frequently used for the identification of parentage, kinship and other forensic purposes, but are also applied in the monitoring of hematopoietic chimerism in patients after allogeneic stem cell transplantation. The suitability of these markers for chimerism analysis, however, depends on different factors, which needs to be considered before recommending an individual marker or a panel of markers for chimerism characterization. It is mandatory for the marker used that an informative for example recipient-specific allele differs in size from the donor alleles by at least one or two repeat units. To accurately describe the relation between donor and recipient alleles, the Eurochimerism Consortium (Appendix A) has developed a proposal for a nomenclature, which provides a uniform identification system of STR alleles applicable for chimerism analysis, which is presented in this issue. ${ }^{6}$ The size of a defined allele can show some degree of variation due to the equipment used for fragment separation, but the inclusion of known reference samples in the analysis can eliminate this problem.

Many STR loci consist of simple repeats, that is, the repeat units are constant throughout the repeat region. For example, the microsatellite loci D3S1768, D19S253 and D10S2325 have simple tetra - or pentanucleotide repeats. The motif structure and number of repeats in selected alleles of these STR loci are shown in Table 1. Although the majority of STR loci are assumed to have simple repeat units based upon their Genome DataBase (GDB) reference sequence, a considerable number of STR loci display variability within the repeats, which may affect the allele assignment. We have investigated the composition of the STRs by sequence analysis and concluded that the existence of such STR loci is a greatly underestimated phenomenon. The so-called compound STR markers consist of repeat motifs displaying uniform length, but variable sequences within the repeat units (e.g. D2S1360 and D12S1064, Table 1). Complex repeats vary in their motif composition (tri-, tetra- and pentanucleotide repeats) and may also contain sequence polymorphisms within individual repeat units, while certain motifs within the remaining repeat units are constant (e.g. P450CYP19, D8S1132, D9S1118 and D12S391, Table 1). The so-called hypervariable complex repeats (e.g. D17S1290, SE-33, D11S554, D7S1517 and MYCL1, Table 1) consist of variable and constant repeat structures. For example, the variability in MYCL1 is determined by the number of tetra- [GAAA] and penta- [GAAAA] nucleotide repeats interspersed between different constant repeat units (Figure 1). D11S554 shows an even higher degree of complexity represented by the constant units $[\mathrm{AAAG}]_{3},[\mathrm{AAAGG}]_{1}$ and $[\mathrm{AAAAA}]_{1}$ combined with a variable number of $[A G]_{n}$ and $[A A A G]_{n}$ motifs and the presence or absence of a number of other variable repeat units. Interestingly, two alleles of this locus, both displaying a fragment length of $220 \mathrm{bp}$, are composed of a different combination of variable repeats (Figure 2). Similarly, the presence of homozygosity was identified by fragment length analysis in a patient sample analyzed by the marker D7S1517, which revealed a single $196 \mathrm{bp}$ allele corresponding with 25 tetranucleotide repeat units (Figure 3a). Sequence analysis, however, showed heterozygosity by revealing the presence of two alleles, as indicated by different nucleotides at certain positions in the sample sequence (Figure 3b). Among six selected patient samples tested by fragment length analysis of the D7S1517 marker, two were identified as homozygous for a $173 \mathrm{bp}$ fragment and four for a196 bp fragment. While the samples displaying the $173 \mathrm{bp}$ fragment were also homozygous at the sequence level, only one of the samples showing the $196 \mathrm{bp}$ fragment was confirmed to be homozygous by sequencing. In the remaining $196 \mathrm{bp}$ fragments, which were demonstrated to be heterozygous by sequence analysis, as many as five different alleles were identified (Figure 4). The examples of hypervariable complex STR markers shown, including SE-33, D11S554, D7S1517, D17S1290 and MYCL1, contain a variety of repeat unit structures. Sequence analysis of numerous samples identified constant and variable units within the repeat sequences. In STR markers with simple repeat structure, the fragment length is indicative of the number of (e.g. tetranucleotide) repeat units, regardless of nucleotide sequence variability and alignment of the repeat blocks. Constant regions within repeats do not affect the allele assignment. Complex repeats, however, contain a variable number of di-, tri-, tetra-, penta-, octanucleotide and other repeat motifs, which make the repeat unit identification and the allele assignment difficult. Although size differences between alleles are useful for the identification of individual human specimens, the actual alleles can only be determined by the analysis of repeat unit polymorphisms using DNA sequencing.

Different fragment sizes of STR alleles reflect only a part of the variability among individuals but do not identify the gene polymorphism itself in all instances. Allele frequencies based upon fragment size analysis are useful for the prediction of informative constellations in forensic medicine and in the monitoring of chimeric status after stem cell transplantation. But, when the actual sequence-defined alleles are considered, statistics based on homozygosity frequencies determined by fragment analysis can be erroneous. Thus, in forensic analysis, the presence of homozygous STRs, with identical fragment sizes, should be interpreted with care. Despite the high sequence variability within repeat motifs, applicability of STR markers in chimerism analysis is not affected. Informative markers for the monitoring of chimerism after stem cell transplantation can be selected on the basis of allele sizes alone, regardless of the complexity of repeat motifs. 
Table 1 Motif structure and number of repeats of the STR loci

\begin{tabular}{|c|c|c|c|}
\hline Marker & Acc.nr. & Chr. & Repeat \\
\hline D3S1768 & G08287 & 3 & {$[\mathrm{AGAT}]_{12}$} \\
\hline D19S253 & L13122 & 19 & {$[\mathrm{ATAG}]_{13}$} \\
\hline D10S2325 & G08790 & 10 & {$[\mathrm{TCTA}]_{12}$} \\
\hline P450CYP19 & M30798 & 15 & {$[T A T \Pi]_{1}[T A T]_{8}$} \\
\hline D2S1360 & G08130 & 2 & {$[\text { TATC }]_{9}\left[\right.$ TGTC $_{9}\left[\mathrm{TATC}_{5}[\mathrm{TCT}]_{2}\right.$} \\
\hline D12S1064 & G08952 & 12 & {$\left[\right.$ TATC $_{13}[\text { TGTC }]_{1}[\text { TATC }]_{1}[\text { ATCT }]_{2}$} \\
\hline D8S1132 & G08685 & 8 & {$[\mathrm{TCTA}]_{9}[\mathrm{TCA}]_{1}[\mathrm{TCTA}]_{9}$} \\
\hline D9S1118 & G08752 & 9 & {$[\mathrm{TATC}]_{8}[\mathrm{ATC}]_{1}[\mathrm{TATC}]_{3}$} \\
\hline D12S391 & G08921 & 12 & {$[\mathrm{GATA}]_{4}[\mathrm{GAT}]_{1}[\mathrm{GATA}]_{8}[\mathrm{GACA}]_{6}$} \\
\hline D7S1517 & L30761 & 7 & {$[\mathrm{GAAA}]_{9}[\mathrm{GAAACAAACAAA}]_{1}[\mathrm{CAAAGAAAGAAA}]_{1}[\mathrm{CAAA}]_{1}[\mathrm{GAAA}]_{1}$} \\
\hline D17S1290 & G07956 & 17 & {$[\text { TAGA }]_{4}[T G A]_{2}[\text { TAGATATA }]_{3}[\text { TAGA }]_{13}$} \\
\hline SE-33 & ACTBP8 & 6 & $\begin{array}{l}{[\mathrm{AAAG}]_{4}[\mathrm{AGACAAAG}]_{1}[\mathrm{AG}]_{2}[\mathrm{TTAG}] 1[\mathrm{AAAG}]_{3}[\mathrm{AG}]_{6}[\mathrm{AAAG}]_{1}[\mathrm{GAAG}]_{3}[\mathrm{AAAAAGAAAG}]_{2}} \\
{[\mathrm{AAAGAGAAAAAA}]_{2}[\mathrm{AAAG}]_{9}[\mathrm{AAAAAGAAA}]_{1}[\mathrm{AAAG}]_{13}[\mathrm{GAAGA}]_{1}}\end{array}$ \\
\hline D11S554 & M87277 & 11 & {$[\mathrm{AAAG}]_{3}[\mathrm{AG}]_{4}[\mathrm{AAAGG}]_{1}[\mathrm{AG}]_{1}[\mathrm{AAAGG}]_{1}[\mathrm{AAAG}]_{4}[\mathrm{AA}]_{1}[\mathrm{AAAG}]_{6}[\mathrm{AAGG}]_{14}[\mathrm{AAGAAA}]_{1}[\mathrm{AG}]_{3}$} \\
\hline Mycl-1 & X63900 & 1 & {$[\mathrm{GAAA}]_{14}[\mathrm{GAAAA}]_{2}[\mathrm{GAAAAAAAA}]_{1}[\mathrm{GAAAA}]_{4}[\mathrm{GAAAAAA}]_{1}[\mathrm{GAAAA}]_{2}[\mathrm{GAAA}]_{1}$} \\
\hline
\end{tabular}

$192[\text { GAAA }]_{2}[\text { TAAA }]_{1}[\text { GGAA }]_{1}[\text { GA }]_{3}[\text { GAAA }]_{1}[\text { GA }]_{1}[\text { GAAA }]_{14}[\text { GAAAA }]_{2}[\text { GAAAAAAAA }]_{1}[\text { GAAAA }]_{4}[\text { GAAAAAA }]_{1}[\text { GAAAA }]_{2}[\text { GAAA }]_{1}$

$191\left[\mathrm{GAAA}_{2}[\mathrm{TAAA}]_{1}[\mathrm{GGAA}]_{1}[\mathrm{GA}]_{3}[\mathrm{GAAA}]_{1}[\mathrm{GA}]_{1}[\mathrm{GAAA}]_{15}\left[\mathrm{GAAAA}_{2}[\text { GAAAAAAAA }]_{1}[\mathrm{GAAAA}]_{3}[\text { GAAAAAA }]_{1}[\text { GAAAA }]_{2}[\text { GAAA }]_{1}\right.\right.$

Figure 1 Identification of sequence variation of repeat units in MYCL1.

\begin{tabular}{|c|c|c|c|c|c|c|c|}
\hline 199 & {$[\mathbf{A A A G}]_{3}$} & {$[\mathrm{AG}]_{9}$} & {$[\mathrm{AAAGG}]_{1}$} & [AAAG] $_{14}$ & {$[\mathbf{A A A A A G}]_{1}$} & & [AAAAA] \\
\hline 203 & {$[\mathrm{AAAG}]_{3}$} & {$[\mathrm{AG}]_{9}$} & {$[\mathrm{AAAGG}]_{1}$} & {$[\mathbf{A A A G}]_{15}$} & {$[\mathbf{A A A A A G}]_{1}$} & & [AAAAA] \\
\hline 207 & {$[\mathrm{AAAG}]_{3}$} & {$[\mathrm{AG}]_{9}$} & {$\left[\mathrm{AAAGG}_{1}\right.$} & {$[\mathbf{A A A G}]_{16}$} & {$[\mathrm{AAAAAG}]_{1}$} & & [AAAAAA] \\
\hline 220 & {$[\mathrm{AAAG}]_{3}$} & {$[\mathrm{AGG}]_{4}$} & {$[\mathrm{AAAGG}]_{1}$} & {$[\mathrm{AG}]_{3}[\mathrm{AAAGG}]_{1}[\mathrm{AAAG}]_{3}$} & {$[\mathbf{A A A G G}]_{2}[\mathbf{A A A G}]_{14}[\mathbf{A A A A A G}]_{1}$} & & [AAAAAA] \\
\hline 220 & {$[\mathrm{AAAG}]_{3}$} & {$[\mathrm{AGG}]_{7}$} & {$[\mathrm{AAAGG}]_{1}$} & {$[\mathbf{A A A G}]_{1}[\mathbf{A G}]_{1}$} & {$[\mathbf{A A A G}]_{20}$} & {$[\mathrm{AG}]_{1}$} & [AAAAAA] \\
\hline 229 & {$[\mathbf{A A A G}]_{3}$} & {$[\mathrm{AGG}]_{4}$} & {$[\mathrm{AAAGG}]_{1}$} & {$\left[\mathrm{AG}_{1}\left[\mathrm{AAAGG}_{1}[\mathrm{AAAG}]_{4}\right.\right.$} & {$[\mathbf{A A A G G}]_{2}[\mathbf{A A A G}]_{17}$} & {$[\mathrm{AG}]_{1}$} & [AAAAAA] \\
\hline
\end{tabular}

Figure 2 Identification of sequence variation of repeat units in D11S554.

a
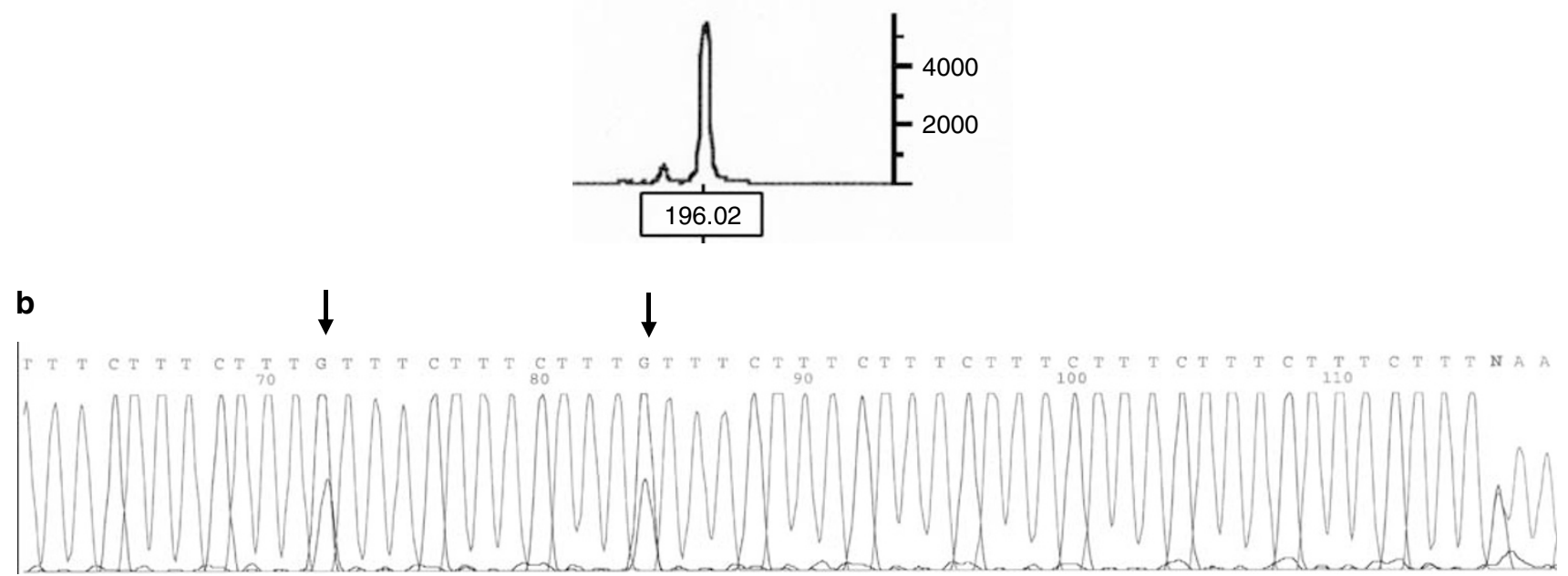

Figure 3 (a): Homozygosity for D7S1517- 196 bp allele by fragment analysis. (b): Sequence analysis of this fragment (reverse complement), arrows point to heterozygous ' $\mathrm{C} / \mathrm{G}^{\prime}$ positions in the sequence revealed by two different repeat structures within the sequence with the same allele length. Two repeat units were identified: GAAA and CAAA. 


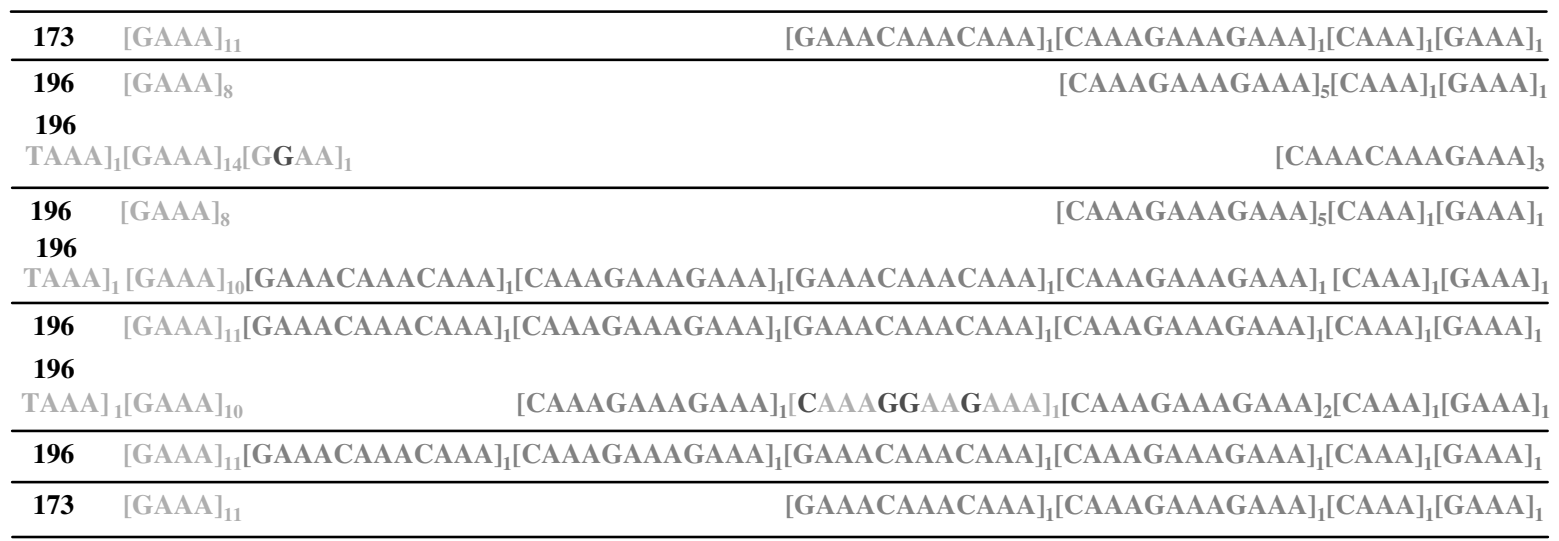

Figure 4

Identification of sequence variation of repeat units in D7S1517.

Moreover, as outlined in the manuscript describing the introduction of the RSD code by the Eurochimerism Consortium, ${ }^{6}$ the variability within STR repeat motifs has impact on the proposed nomenclature of microsatellite markers eligible for chimerism analysis.

MGJ Tilanus on behalf of the Eurochimerism Consortium ${ }^{1}$ Department of Pathology, Heidelberglaan, Utrecht The Netherlands E-mail: m.tilanus@umcutrecht.n ${ }^{1}$ The Eurochimerism consortium includes 12 leading diagnostic centers from 10 European countries (listed at the end of the paper) and is supported by a grant from the European Commission within the fifth Framework Program. The study on variability within repeats has been performed at the Department of Pathology, Utrecht, The Netherlands by $J H$ van Loon, $L$ de Vries, RA de Weger and MGJ Tilanus

\section{References}

1 Lins A, Sprecher C, Puers C, Schumm J. Multiplex sets for the amplification of polymorphic short tandem repeats loci;

\section{Appendix A}

EUROCHIMERISM consortium:

Thomas Lion (Coordinator), Franz Watzinger, Sandra Preuner,

Children's Cancer Research Institute, Vienna, AUSTRIA;

Peter Bader (Deputy coordinator), Hermann Kreyenberg, Dept. of Pediatrics, University Frankfurt, GERMANY;

Hélène Cavé, Cécile Acquaviva, Hopital Robert Debré, Paris FRANCE;

Mark Lawler, Karen Molloy, Mireille Crampe, St. James's Hospital, Dublin, IRELAND;

Andrea Biondi, Federica Colnaghi, Dept. of Pediatrics, University Milano, Monza, ITALY; Jacques van Dongen, Mirjam van der Burg, Dept. of Immunology, University Rotterdam, NETHERLANDS; silver stain and fluorescent detection. Biotechniques 1996; 20: 882-889.

2 Oberkircher A, Strout M, Herzig G, Fritz P, Caligiuri M. Description of an efficient and highly informative method for the evaluation of hematopoietic chimerism following allogeneic bone marrow transplantation. Bone Marrow Transplant 1995; 16: 695-702.

3 van Blokland $\mathrm{W}$, Ignatiadis MG, Bosboom EC, Verdonck LF, van Wichen DF, Tilanus MGJ et al. An optimal microsatellite analysis for donor and recipient cells after bone marrow transplantation. In: Charron D (ed). Genetic Diversity of HLA, Functional and Medical Implication. EDK: Paris, France, 1997, pp 586-588.

4 Kreyenberg H, Möhrle S, Hoelle W, Niethammer D, Bader P. Chimerism analysis using fluorescent based PCR analysis of STR after capillary electrophoresis - The Tuebingen experience. Leukemia 2003; 1: 237-240.

5 Lion T. Summary: reports on quantitative analysis of chimerism after allogeneic stem cell transplantation by PCR amplification of microsatellite markers and capillary electrophoresis with fluorescence detection. Leukemia 2003; 17: 252-254.

6 Watzinger F, Lion T, Steward C. The RSD code: Proposal for a nomenclature of allelic configurations in STR-PCR based chimerism testing after allogeneic stem cell transplantation. Leukemia, this issue.

Giuseppe Saglio, Anna Serra, Dept of Clin. and Biol. Sciences, University Turin, ITALY;

Marcos González-Díaz, Miguel Alcoceba, Dept. of Hematology, University Salamanca, SPAIN;

Gisela Barbany, Monica Hermanson, Clinical Genetics, University Hospital, Uppsala, SWEDEN; Eddy Roosnek, Dept. of Transplant Immunology, University Geneva, SWITZERLAND;

Marcel Tilanus, Roel de Weger, Joyce van Loon, Lianne de Vries, Dept. of Pathology, University Medical Center, Utrecht, NETHERLANDS

Colin Steward, Royal Hospital for Children, Bristol, UNITED KINGDOM;

John Harvey, H\&I Laboratory, National Blood Service, Bristol, UNITED KINGDOM 